\title{
ANÁLISE DA ABORDAGEM SINGLE POINT RANS PARA O ESCOAMENTO DO TIPO JATO LIVRE AXISSIMÉTRICO E INCOMPRESSÍVEL
}

\author{
Gisely Luzia Ströher ${ }^{1}$ \\ Joel Fernando Nicoleti ${ }^{2}$ \\ Cláudia Regina de Andrade $e^{3}$ \\ Edson Luiz Zaparoli ${ }^{4}$ \\ Gylles Ricardo Ströher 5
}

\begin{abstract}
Resumo: Foram efetuadas diversas simulaçôes numéricas para o escoamento do tipo jato livre axissimétrico, incompressível e turbulento, no intuito de avaliar numericamente as afirmaçóes de Taulbee (1989), George (1989) e George e Davidson (2004) as quais convergem na afirmação de que a modelagem single-point RANS (Reynolds-averaged Navier-Stokes) não possui a física necessária para contabilizar a dependência assintótica da origem do jato no seu desenvolvimento. $\mathrm{O}$ objetivo principal deste trabalho limita-se a mostrar alguns resultados, sem tentar solucionar tal afirmação. Adicionalmente é apresentada uma breve análise da influência das condiçóes de origem do jato sobre a regiáo completamente desenvolvida do escoamento. Os resultados das simulaçóes corroboram a afirmaçáo dos referidos autores e também indicam que náo há um conjunto geral de constantes dos modelos de turbulência que se apresente universalmente válido. Foi verificado que a regiáo completamente desenvolvida mostra-se dependente não apenas da quantidade de momento na saída do bocal, conseqüentemente, os jatos atingem diferentes estados autossimilares em funçáo dos detalhes de sua origem, contrapondo a hipótese clássica da autossimilaridade.
\end{abstract}

Palavras-chave: Jato Livre, Dinâmica dos Fluidos Computacional, Autossimilaridade, Modelos de Turbulência Rans.

Abstract: In this study, we performed several numerical simulations for the axisymmetric free jet flow, incompressible and turbulent, in order to evaluate the assertions from Taulbee (1989), George (1989) and George and Davidson (2004), which converge in the statement that the single-point RANS (Reynoldsaveraged Navier-Stokes) modeling does not possess the necessary physics to account for the asymptotic dependence of the origin of the jet in its development. The main goal of the present study is limited to show some results, without attempting to solve this assertion. Additionally we present a brief analysis of the influence of the jet origin conditions on the fully developed flow region. The results from simulations corroborate the assertion of these abovementioned authors and also indicate that there is no general set of constants of turbulence models that is universally valid. We verified that the fully developed region is dependent not only on the amount of momentum at the nozzle exit, consequently, the jets reach different self-similar states in function of the details of its origin, contrasting the classical self-similarity hypothesis.

Keywords: Free Jet, Computational Fluid Dynamics, Self-Similarity, Rans Turbulent Models

\footnotetext{
1 Universidade Tecnológica Federal do Paraná.

2 Universidade Tecnológica Federal do Paraná.

3 Instituto Tecnológico de Aeronáutica.

4 Instituto Tecnológico de Aeronáutica.

5 Universidade Tecnológica Federal do Paraná.
} 


\section{INTRODUÇÁO}

O escoamento do tipo jato livre ocorre quando um fluido é expandido por meio de um bocal ou orifício para um ambiente no qual o escoamento não é diretamente afetado por um contorno fixo.

O jato livre tem sido objeto de extensos estudos experimentais e de simulaçóes numéricas nas últimas quatro décadas (Chang, 2002; Klein et al., 2003) devido às amplas aplicaçóes deste tipo de escoamento nas mais diversas áreas, como: lubrificação, injetores de combustível, processos de combustão industrial e de aeronaves.

A estrutura do escoamento de um único jato livre turbulento incompressível é ilustrada na Figura 1.

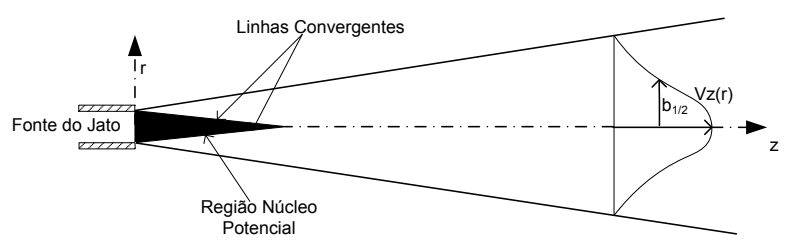

Figura 1. Estrutura do escoamento de um jato livre circular.

Após a saída do fluido do bocal, tem início a regiáo núcleo potencial, em que o fluido é liberado possuindo a maior velocidade, sendo a sua componente axial constante. À medida que as camadas de cisalhamento se encontram, estreitando a regiáo de núcleo potencial, o jato inicia a transição para o regime completamente desenvolvido.

As duas linhas convergentes na Figura 1 representam o crescimento das camadas cisalhantes, e a distância entre o bocal até a intersecção destas duas linhas é chamada de comprimento da região de núcleo potencial.

A região de transiçấo é a região intermediária entre o término da regiáo de núcleo potencial e o início da regiáo completamente desenvolvida. Após a passagem pela região de transição, o jato atinge a região completamente desenvolvida ou autossimilar, onde a velocidade média axial do jato não é mais constante.

Existe grande discussão a respeito das características da região completamente desenvolvida; nesta, os perfis de velocidade e das tensóes de Reynolds escalonados corretamente colapsam em uma única curva do tipo Gaussiana.

Em coordenadas cilíndricas para um jato circular axissimétrico, o perfil radial autossimilar de uma variável $\Theta$ pode ser dado por:

$$
\Theta(z, r)=\Theta_{c}(z) f(\eta)
$$

$\Theta_{c}(z)$ é o máximo local de $\Theta(z, r)$, que é encontrado na linha de centro do jato $(\mathrm{z}, \mathrm{r}=0)$; a função $f$ contabiliza toda a variação radial, sendo que $f(0)=1$.

Aplicando a Equação (1) para a componente axial da velocidade e para as tensóes de Reynolds obtêm-se, respectivamente:

$$
\begin{aligned}
& V_{z}(z, r)=V_{z c}(z) f_{1}(\eta) \\
& -\overline{v_{z} v_{r}}=R_{c}(z) f_{2}(\eta)
\end{aligned}
$$

Sendo $\eta=r / \delta$ e $\delta=\delta(z)$, as funçóes $f_{I}(\eta)$ e $f_{2}(\eta)$ contabilizam toda a variaçáo radial, e a existência de ambas implica que os perfis de velocidade e das tensóes de Reynolds a cada estação do escoamento do jato podem colapsar em um único perfil (George, 1989). As variações axiais são contabilizadas pelas funçóes $V_{c}(z), R_{c}(z)$ e $\delta(z)$.

A variação de $V_{c}(z)$ ao longo do jato pode ser descrita por:

$$
\frac{V_{c}(z)}{V_{o}}=K_{1}\left(\frac{d_{\varepsilon}}{z-z_{01}}\right)
$$

em que $z_{01}$ é a origem virtual, $K_{1}$ é a constante de decaimento do jato, $d_{\varepsilon}$ é o diâmetro efetivo do bocal e $V_{o}$ é a média da componente axial de velocidade no plano de saída do jato.

O espalhamento do jato é tipicamente caracterizado pela variável $b_{1 / 2}$, meio raio, definido como a localização na coordenada radial em que a componente axial da velocidade é a metade da velocidade da linha de centro do jato, ou seja, a posição $r$ tal que $V_{z}\left(r, z_{i}\right)=0,5 V_{z}\left(r=0, z_{i}\right)$.

Também na regiáo autossimilar, em geral, $b_{1 / 2}$ varia linearmente $\operatorname{com} z$, da seguinte forma:

$$
\frac{b_{1 / 2}}{d_{\varepsilon}}=K_{2}\left(\frac{z-z_{02}}{d_{\varepsilon}}\right)
$$

em que $K_{2}$ é a taxa de espalhamento do jato e $z_{02}$ representa a origem virtual do jato. $\mathrm{O}$ diâmetro efetivo $d_{\varepsilon}$ é definido como: 


$$
d_{\varepsilon}=\frac{2 M_{0}}{\sqrt{\pi \rho_{\infty} J_{0}}}
$$

em que:

$$
\begin{aligned}
& M_{0}=\int_{0}^{D / 2} 2 \pi \rho_{0} U_{0} r d r \\
& J_{0}=\int 2 \pi \rho_{0} U_{0}^{2} r d r
\end{aligned}
$$

$M_{0}$ é o fluxo de massa e $J_{0}$ é a quantidade de movimento, ambos na saída do jato; $\rho_{0}$ é a massa específica do fluido na saída do jato; $\rho_{\infty}$ é a massa específica do fluido ambiente. $\mathrm{O}$ conceito de diâmetro efetivo foi utilizado no presente trabalho, como utilizado por outros autores (Thring e Newby, 1953; Beer et al., 1962; Becker et al., 1967; Dowling e Dimotakis, 1990; Pitts, 1991A; B; Richards e Pitts, 1993), para contabilizar os efeitos da massa específica do fluido inicial e o perfil de velocidade média do jato.

Até meados da década de 1980 foi amplamente aceita a hipótese clássica da autossimilaridade, que estabelecia que na região completamente desenvolvida todos os jatos atingiriam o mesmo estado autossimilar, independentemente dos detalhes de sua origem, e dependeriam apenas da quantidade de momento na saída do bocal $\left(\mathrm{J}_{0}\right)$ (Towsead, 1976). Consequentemente, os parâme$\operatorname{tros} z_{01} z_{02}, K 1$ e $K 2$ do jato e os perfis escalonados (Equaçóes 2 e 3) de todos os jatos seriam idênticos, ou seja, acreditava-se que a turbulência esquecia suas origens.

Através de estudos experimentais, concluiu-se que o escoamento escalonado (Equaçóes 2 e 3 ) de um jato turbulento atinge o estado autossimilar em uma região suficientemente longe do bocal (Dowling e Dimotakis, 1990). Entretanto, existia considerável confusão acerca dos valores que descrevem o escoamento escalonado, se é universal ou não, por exemplo, a variação entre trabalhos experimentais de aproximadamente $40 \%$ para o valor da taxa de espalhamento do jato $(K 2)$.

No final da década de 80 , a validade da hipótese clássica da autossimilaridade universal foi questionada analiticamente por George (1989) ao sugerir que o escoamento turbulento pode ter múltiplos estados autossimilares determinados unicamente por suas condiçóes iniciais.

George (1989) realizou um estudo analítico com o objetivo de prover uma modelagem para a possível relação entre a autossimilaridade e as condiçôes iniciais. $\mathrm{O}$ autor argumentou que a aparente discrepância entre os valores experimentais de $z_{01,} z_{02,} K 1$ e $K 2$ não se situa nos experimentos em si, nem no conceito de autossimilaridade, mas particularmente no modo limitado como a análise da autossimilaridade clássica havia sido concebida.

Outros autores também discutiram em detalhe o assunto, como Moser et al. (1998) e Johansson et al. (2003). Em todos esses estudos, os resultados foram semelhantes: a equação de momento escalonada implica que a velocidade média e os perfis das tensôes de Reynolds corretamente escalonados são sempre independentes das condiçóes iniciais (ou origem). No entanto os parâmetros de escalonamento náo são independentes e outros perfis, como o da energia cinética turbulenta, também não seriam universais.

Desta forma, o resultado do colapso de alguns perfis (como o da velocidade média) não pode ser associado com o fato de a turbulência não manter a dependência das condiçôes de origem. Ao invés disto, é um resultado artificial da escolha das escalas e da equação da quantidade de movimento. Entretanto, foi precisamente essa universalidade dos perfis médios que levou a comunidade científica a aceitar equivocadamente a ideia de independência das condiçóes de origem, até poucos anos atrás.

Como comentam Mi et al. (2001 a) e George e Davidson (2004), não há um estudo definitivo disponível na literatura que relata o impacto das condiçôes iniciais sobre jatos turbulentos. Uma abordagem aplicável para verificar a influência das condiçóes de origem sobre a evolução do escoamento é a dinâmica dos fluidos computacional. Entretanto, discute-se se os modelos single-point RANS (Reynolds Averaged Navier Stokes) de turbulência seriam capazes de contabilizar os efeitos da origem do jato sobre a evoluçáo do escoamento turbulento, sobretudo na região autossimilar.

Com base no exposto acima, o objetivo principal deste trabalho é avaliar numericamente as afirmaçóes de Taulbee (1989), George (1989) e George e Davidson (2004) as quais indicam que a modelagem RANS não possui a física necessária para contabilizar os efeitos da origem do jato. Adicionalmente, pretende-se realizar uma breve análise da influência das condiçóes de origem do jato sobre a regiáo completamente desenvolvida (autossimilar). 


\section{MODELAGEM MATEMÁTICA}

O problema físico em estudo é um jato de ar livre circular proveniente de um bocal de diâmetro $\mathrm{D}$ que se expande para um ambiente no qual se encontra ar inicialmente estagnado. $\mathrm{O}$ escoamento é em regime permanente, axissimétrico, incompressível, isotérmico e turbulento.

Com as hipóteses citadas acima, as equaçóes de Navier Stokes com a média de Reynolds podem ser escritas da seguinte forma:

$$
\begin{aligned}
& \nabla \cdot U=0 \\
& U \cdot \nabla U=-(1 / \rho) \nabla P+\nabla \cdot\left[\left(v+v_{t}\right) \nabla U\right]
\end{aligned}
$$

em que $\rho$ é a massa específica do fluido, $U$ é o vetor da velocidade média de Reynolds, $P$ é a pressão média de Reynolds, $v$ é a viscosidade molecular dinâmica e $v_{t}$ é a viscosidade turbulenta.

Para o fechamento das Equaçóes (9) e (10) foi utilizado inicialmente o modelo de duas equaçóes $k-\varepsilon$ de Shih et al. (1995), comumente chamado de modelo $k-\varepsilon$ Realizável. Este modelo foi escolhido por apresentar bons resultados, em relação a outras versôes dos modelos $k-\varepsilon$, para o problema de jato livre circular turbulento incompressível (Shih et al. 1995; Ströher et al., 2008).

\subsection{DOMÍNIO COMPUTACIONAL E CONDIÇÓES DE CONTORNO}

A geometria em consideração, as condições de contorno e o sistema de coordenadas são mostrados na Figura 2. O escoamento de jato livre foi resolvido utilizando um domínio bidimensional axissimétrico. Em simulaçôes prévias a dimensão de domínio computacional de $50 D$ e $10 D$, nas coordenadas espaciais $z$ e $r$, respectivamente, mostrou-se suficiente para garantir as hipóteses assumidas nas condiçóes de contorno $\left(P=P_{a m b}\right)$. Tais dimensôes são superiores às tipicamente encontradas na literatura para simulação de jatos livres (Mani et al., $1997(z / D=30, r / D=10)$, Keysar e Degani, 2004 (jato supersônico, $z / D=40, r / D=$ 10), Senesh e Babu, 2005 (jato subsônico, $z / D=$ $40, r / D=10$ e jato supersônico $z / D=20, r / D=$ 5)).

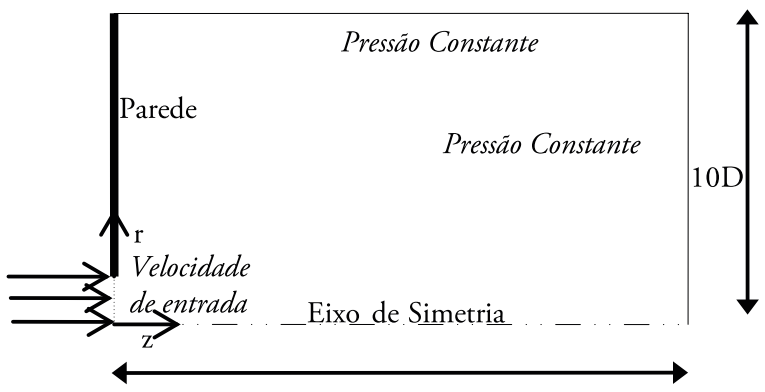

Figura 2: Domínio Computacional (desenho sem escala).

Com referência a Fig.2 o perfil de velocidade $\mathrm{v}(\mathrm{r})$ e os valores das propriedades turbulentas, $\mathrm{k}$ e $\varepsilon$ foram especificados para cada caso, estas duas últimas variáveis foram relacionadas com a intensidade de turbulência e o diâmetro hidráulico em conformidade com Versteeg e Malalasekera (1995).

Considerou-se que o escoamento se estende suficientemente ao longo do domínio, de forma que, a pressão é a mesma que a do ambiente.

\subsection{SOLUÇÃO NUMÉRICA}

O campo de escoamento foi obtido através da solução numérica das equações de Navier Stokes com média de Reynolds e das equaçóes dos modelos de turbulência disponíveis no software Fluent 6.3. Foi utilizado o método segregado com o algoritmo de acoplamento pressão-velocidade Simplec (Vandoormaal e Raithby, 1984), Os termos convectivos foram discretizados e utilizou-se o esquema upwind de segunda ordem.

As soluçōes numéricas foram consideradas convergidas quando o resíduo máximo de todas as equaçóes discretizadas foi menor que $1 \times 10^{-5} \mathrm{e}$ quando a integral da quantidade de momento na direção radial tornou-se independente da coordenada espacial $z$.

Foram geradas três malhas cartesianas uniformes (com 30.000, 61.235 e 99.190 número de células) para o domínio mostrado na Figura 2.

A diferença máxima entre a componente axial da velocidade na linha de centro entre as duas malhas mais refinadas foi menor que $0,5 \%$ para todos os casos; assim, a malha com refinamento intermediário foi utilizada para as simulações numéricas. 


\section{RESULTADOS E DISCUSSÁO}

Inicialmente, realizou-se a comparação entre dados experimentais de um jato livre com os resultados obtidos numericamente. Esta etapa teve como objetivo reproduzir os dados experimentais de Frost e Jambunathan (1996). O trabalho destes autores foi escolhido, pois o perfil de velocidade experimental na saída do jato é plano. Os autores também forneceram a intensidade de turbulência em função do raio do bocal, uma informação valiosa que envolve problemas de jatos livres em regime turbulento, como será apresentado ao longo deste trabalho, e geralmente não é informada devido à dificuldade em mensurá-la. Adicionalmente, a seleção dos dados experimentais baseou-se na disponibilidade de informaçôes que provessem condições de reprodução para a realização da simulação numérica e preferencialmente acompanhada de análise de erros experimentais.

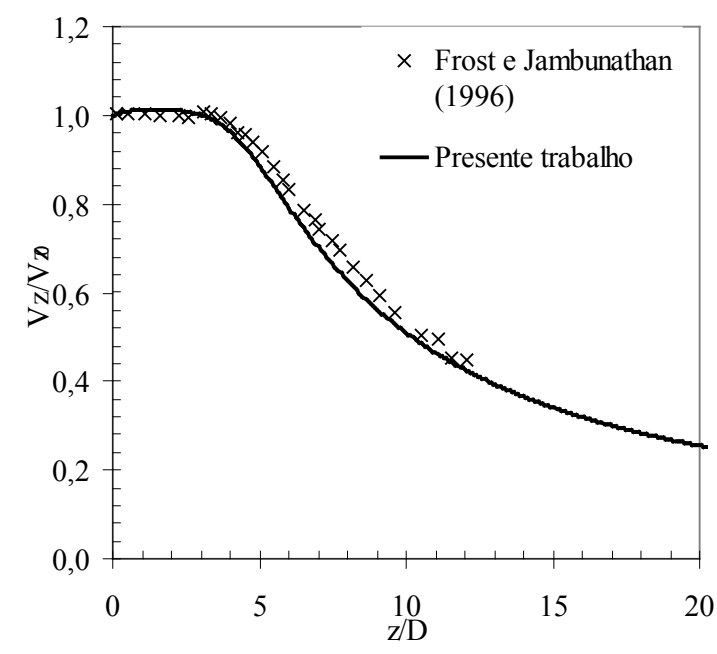

Figura 3: Comparação entre os resultados da simulação numérica diante dos dados experimentais.

$\mathrm{Na}$ Figura 3 é comparado o decaimento da componente axial da velocidade adimensional $\left(\mathrm{V}_{\mathrm{z}} / \mathrm{V}_{\mathrm{z} 0}, \mathrm{~V}_{\mathrm{z} 0}\right.$ = velocidade média na saída do bocal $)$ na linha de centro do jato, a partir da saída do bocal, para um jato livre com número de Reynolds $\left(\mathrm{Re}_{0}=\mathrm{V}_{\mathrm{z} 0} \mathrm{D} v^{-1}\right)$ baseado nas condiçóes no plano de saída do bocal de 22.500 .

No trabalho experimental de Frost e Jambunathan (1996) não são fornecidos os perfis radiais de velocidade para que se possa fazer a comparaçáo entre os resultados numéricos e experimentais. Entretanto, como foi comentado na Seção 1, o perfil da componente axial da velocidade torna-se autossimilar na regiáo completamente desenvolvida quando corretamente escalonado. Desta forma, os perfis obtidos numericamente a partir da componente velocidade axial foram comparados com o perfil escalonado disponível em Schilichting (1979).

Conforme apresentado na Figura 4, os resultados numéricos concordam satisfatoriamente com os dados experimentais.

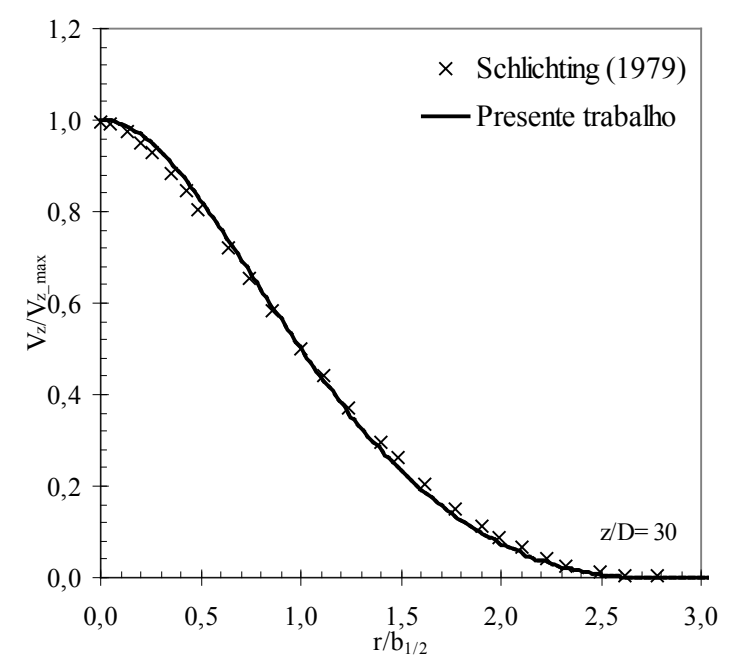

Figura 4: Comparação entre os resultados da simulação numérica diante dos dados de Schlichting (1979).

Como discutido na Seção 2.2, a região desenvolvida tem sido objeto de estudos devido a questão da turbulência esquecer ou não suas origens. Neste sentido, será avaliada a influência das condiçóes de origem do jato nas constantes $z_{01}$, $K 1, z_{02}$ e $K 2$.

As grandezas variadas na origem do jato foram as seguintes:

a) número de Reynolds $\left(R e_{0}\right)$ definido nas condiçôes de origem do jato;

b) intensidade de turbulência no bocal $\left(I_{0}\right)$;

c) influência do perfil de velocidade no bocal.

\subsection{INFLUÊNCIA DO NÚMERO DE REYNOLDS}

Para avaliar a influência das condiçóes de origem do jato, variou-se o número de Reynolds da saída do bocal $\left(R e_{0}\right)$ de 3.000 a 70.000 e foram obtidos os valores de $z_{0}, K_{1}, z_{a}$ e $K_{2}$, que são mostrados nas Figuras 5, 6, 7 e 8, respectivamente. 


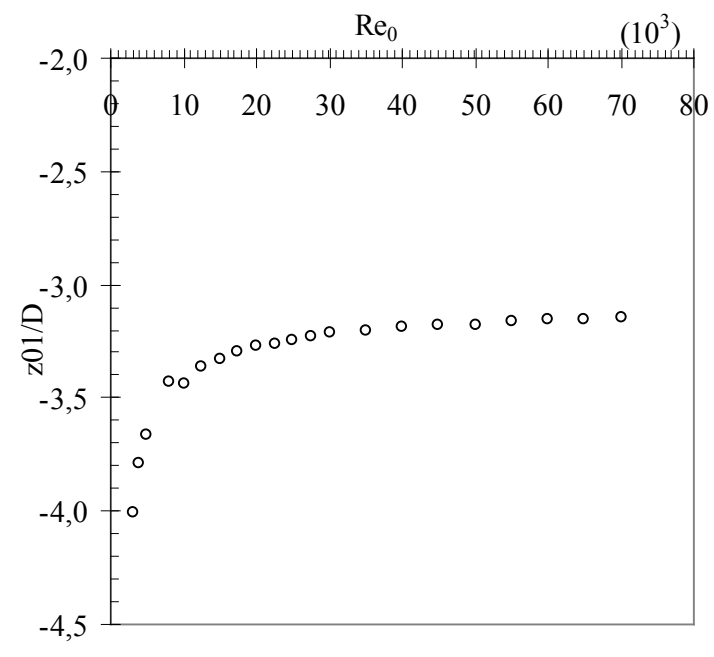

Figura 5: Variação de $z_{01} / D$ em função do número de Reynolds do bocal.

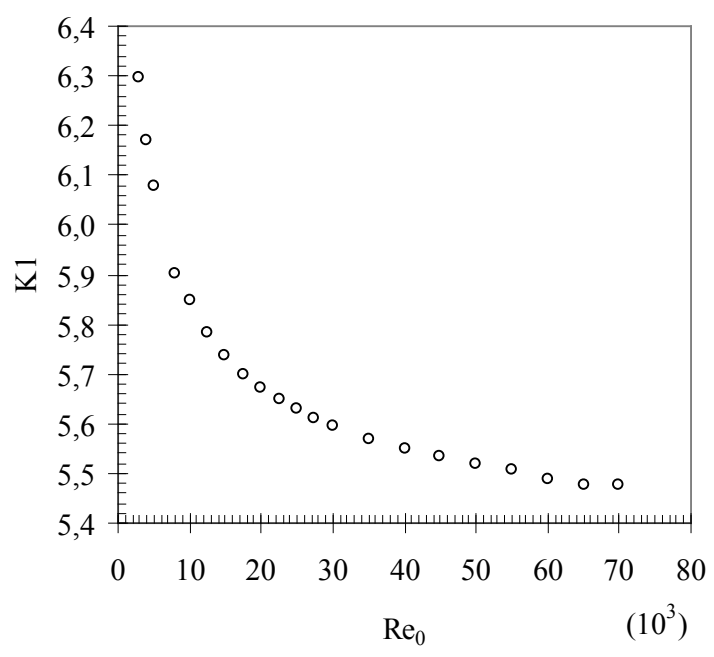

Figura 6: Variação de $K 1 / D$ em função do número de Reynolds do bocal.

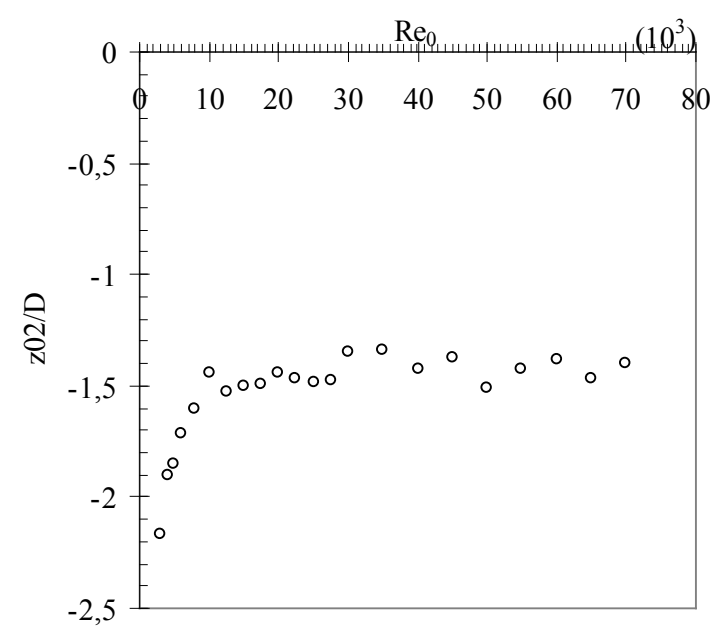

Figura 7: Variação de $z_{02} / D$ em função do número de Reynolds do bocal.

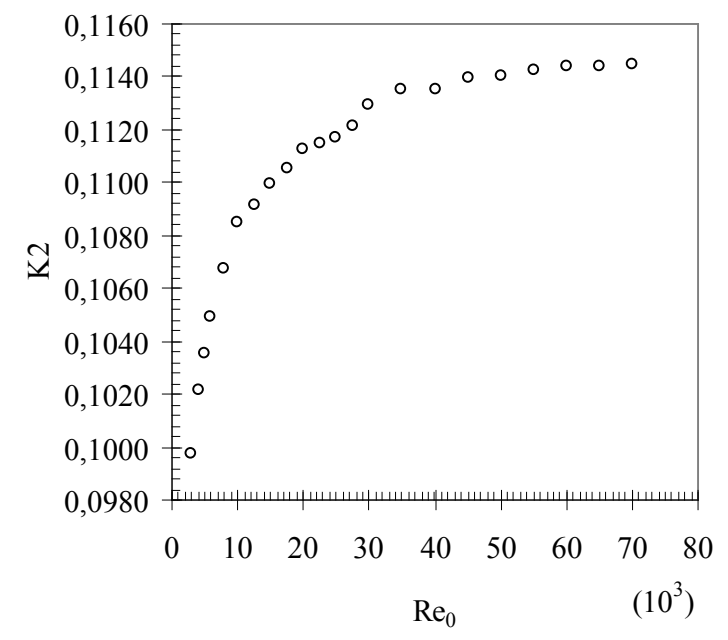

Figura 8: Variação de $K / D$ em função do número de Reynolds do bocal.

Nas Figuras 5 a 8 observa-se que, para números de $R e_{0}$ menores que 10.000 , o escoamento não atingiu completamente o estado autossimilar. Este resultado é semelhante ao obtido com um jato plano (bidimensional) pelos autores Klein et al. (2003) que encontraram forte influência nas características do jato para $R e_{0}$ menores que 6.000.

À medida que o número de Reynolds aumenta é evidente que, a respeito desses quatro parâmetros, o escoamento é próximo de um estado convergido, embora ainda náo atingido, visto que, exceto por $z_{02}$, os parâmetros $K 2$ e $z_{01}$ apresentam comportamento assintótico crescente com o número de Reynolds e decrescente para $K 1$.

$\mathrm{O}$ parâmetro $z_{02}$ para $R e_{0}$ acima de 10.000 não apresentou variação sistemática com $R e_{0}$, resultado semelhante ao obtido por Namer e Ötügen (1988) para o parâmetro $z_{01}$ com um jato plano turbulento.

A revisão na literatura dos valores dos parâmetros $K_{1}, z_{0}, K_{2}$ e $z_{Q}$ para um jato livre axissimétrico é apresentada na Tabela 1, juntamente com os valores de máximo e de mínimo dos quatro parâmetros obtidos no presente trabalho.

A Tabela 1 mostra ampla variaçáo existente em todos os parâmetros avaliados, evidenciando a influência das condiçóes de origem no desenvolvimento do jato. A taxa de espalhamento $\left(K_{2}\right)$, em particular, demonstra ser o parâmetro menos sensível à variação de $R e_{0}$. Segundo Mi et al. (2000a), esta insensibilidade deve-se ao fato de que a variável $b_{1 / 2}$ é definida como a localização radial em que o valor de $\Theta(z, r)$ é igual à metade do seu valor na linha de centro do jato, isto é, $\Theta(z, r)=0,5 \Theta_{c}(z)$ 
, de forma que qualquer erro experimental sistemático contamina igualmente todas as medidas de $\Theta(z, r)$; assim espera-se que as estimativas de $b_{1 / 2}$ sejam menos afetadas por erros experimentais.

Outro resultado importante refere-se à faixa de valores encontrados para a taxa de espalhamento $\left(K_{2}\right)$, mostrada na Figura 8 e explicitada na Figura 9, juntamente com os dados experimentais de Tanaka e Tanaka (1976) e as referências sumarizadas da Tabela 1 . Estes resultados mostram que o modelo $k-\varepsilon$ de Shih et al. (1995) avalia razoavelmente bem a influência das condiçôes de origem do jato sobre a taxa de espalhamento do jato $\left(K_{2}\right)$.

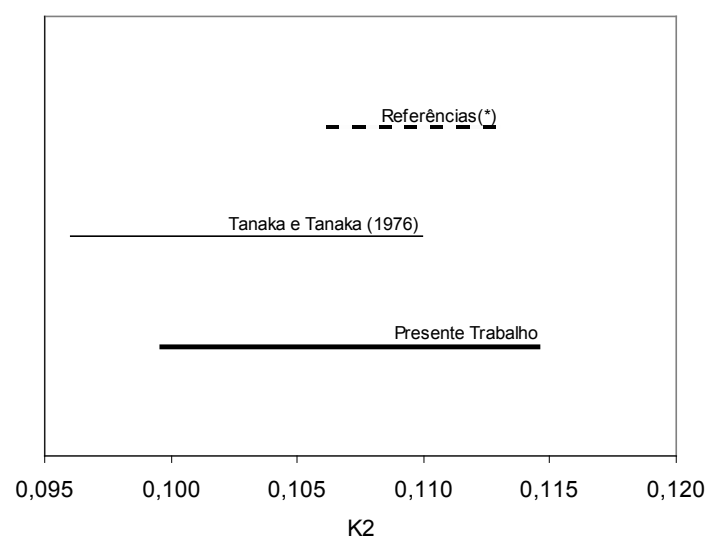

Figura 9: Faixa de Valores para a taxa de espalhamento do jato ( ${ }^{*}$ compilaçáo dos dados da Tabela $1)$.
Dando continuidade ao estudo da influência das condiçóes de origem do jato, foi utilizado o método proposto por Dahm e Dimotakis (1990) para observaçáo da taxa de decaimento da linha de centro do jato. Neste método constrói-se um gráfico de $\chi \Theta_{c} / \Theta_{e}$ versus $\chi$. Os resultados com os dados numéricos do presente trabalho são mostrados na Figura 10.

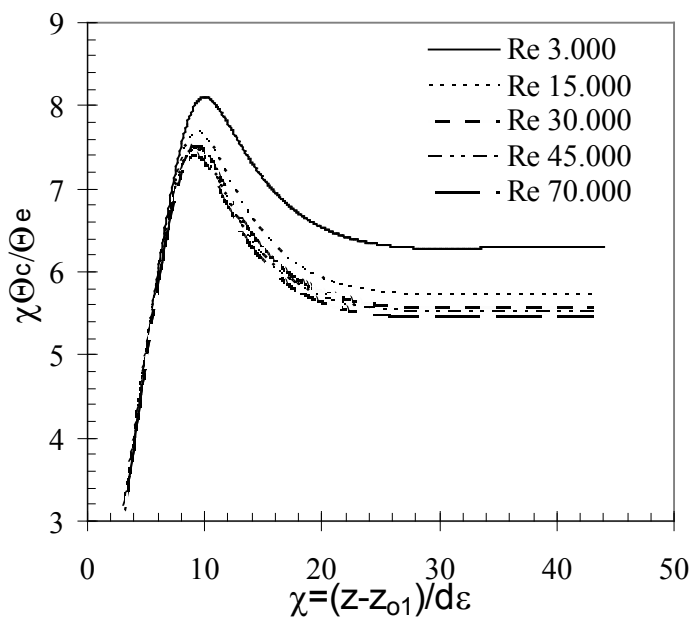

Figura 10: Comparação da variação de $\chi \Theta_{c} / \Theta_{e}$ versus $\chi$ para vários números de Reynolds.

Os dados da forma da Figura 10 indicam que a maioria dos conjuntos de dados tendem a atingir um estado autossimilar como indicado por uma aproximação assintótica a uma linha hori-

Tabela 1 - Valores dos parâmetros $K_{1}, z_{0}, K_{2}$ e $z_{a}$ para um jato livre axissimétrico com perfil de velocidade plano na saída do bocal.

\begin{tabular}{|l|c|c|c|c|c|c|c|}
\hline Autor & $\rho_{e} / \rho_{\infty}$ & $\mathrm{Re}_{0}$ & $\mathrm{~K}_{1}$ & $z_{01} / D$ & $\mathrm{~K}_{2}$ & $z_{02} / D$ & Tipo \\
\hline Becker et al. (1967) & 1,0 & 54.000 & 5,59 & 2,4 & 0,106 & 2,4 & Experimental \\
\hline Wygnanski e Fiedler (1969) & - & 10.000 & 5,7 & 3 & - & - & Experimental \\
\hline McQuaid e Wright (1974) & 1,38 & 10.000 & 4,52 & $-0,01$ & - & - & Experimental \\
\hline Rodi (1975) & - & 87.000 & 5,9 & - & - & - & Experimental \\
\hline Grandmaison et al. (1982) & 1,0 & 27.000 & 5,43 & 1,67 & 0,105 & 1,67 & Experimental \\
\hline So et al. (1990) & 0,64 & 4.300 & 5,51 & 2,0 & 0,107 & 2,0 & Experimental \\
\hline Richards (1993) & 1,552 & 25.000 & 4,76 & $1,6 \pm 3,9$ & 0,113 & 3,6 & Experimental \\
\hline Panchapakese Lumley (1993) & - & 11.000 & 6,1 & - & - & - & Experimental \\
\hline Hussein (1994) & - & 10.000 & 5,8 & 4 & - & - & Experimental \\
\hline Mi (2001) & 0,85 & 16.000 & 4,48 & 3,5 & 0,111 & $-1,0$ & Experimental \\
\hline Presente trabalho & 1,0 & - & - & - & - & - & Numérico \\
& & 70.000 & 6,297 & $-3,15$ & 0,1145 & $-1,396$ & \\
\hline
\end{tabular}


zontal. Entretanto, contrariando o que era esperado da teoria clássica, existe uma variedade de valores assintóticos que tendem ao mesmo perfil para números de Reynolds $\left(R e_{0}\right)$ elevados.

\subsection{INFLUÊNCIA DA INTENSIDADE DE TURBULÊNCIA}

Para avaliar a influência da intensidade de turbulência na saída do bocal $\left(I_{\partial}\right)$ sobre a regiáo autossimilar, três simulaçóes (Casos A, B e C), com diferentes $I_{0}$ foram realizadas com número de Reynolds $\left(R e_{0}\right)$ de 11.250. O nível de intensidade de turbulência atribuído para o Caso $\mathrm{C}$ foi de $1 \%$, valor típico de dados experimentais de um escoamento próximo do regime laminar (Stanley e Sarkat, 2000); já para o Caso A foi atribuído intensidade de turbulência de $17 \%$, que é próximo do que poderia ocorrer em condiçóes turbulentas; para o Caso B, um valor intermediário de $10 \%$.

É importante ressaltar que a manutenção do número de Reynolds $\left(R e_{0}\right)$ fornece praticamente a mesma quantidade de momento $\left(J_{0}\right)$, independentemente do nível de intensidade de turbulência $\left(I_{0}\right)$.

Considerando a quantidade $I_{0}$ a integral do momento pode ser transformada para integral do momento de segunda ordem (Hussein et al., 1994) expressa por:

$$
J_{0,2}=\int_{0}^{D / 2} 2 \pi \rho_{0} U_{0}^{2}\left(1+I_{0}^{2}\right) r d r
$$

A partir da Equação (11), foi calculado $\mathrm{J}_{0,2}$ para os três casos utilizando-se um perfil de velocidade plano na saída do bocal. Os resultados são sumarizados na Tabela 2.

As Figuras 11 e 12 mostram a variaçáo da velocidade adimensional da linha de centro do jato e da meia largura do jato $b_{1 / 2}$ com a distância axial adimensional. Observa-se que $I_{0}$ tem forte influência sobre o jato: quando $I_{0}$ é $1 \%$, o com- primento da região de núcleo potencial, baseada na constância da velocidade adimensional $\left(U / U_{0}\right)$, diminui de $7 D$ para $3 D$. Os parâmetros $K 1, z_{01}$, $K 2$ e $z_{02}$ levantados na regiáo autossimilar para os Casos A, B e C sáo sumarizados na Tabela 2

Os índices 1 e 2 representam momentos de primeira e segunda ordem, respectivamente.

Há diminuição das origens virtuais $\mathrm{z}_{01}$ e $\mathrm{z}_{02}$ com o aumento da intensidade de turbulência, $o$ que indica evoluçáo mais rápida para o comportamento linear característico da região autossimilar do jato.

Com exceção da taxa de espalhamento $\left(K_{2}\right)$, que variou diretamente proporcional com $I_{0}$, Stanley e Sarkar (2000), utilizando Simulação Numérica Direta (SND) para um jato plano, obtiveram a mesma tendência para os outros três parâmetros da regiáo autossimilar, redução de $z_{01}, z_{02}$ e $K_{1}$ com o aumento de $I_{0}$.

Ainda em relação à taxa de espalhamento do jato, Hussain e Zedan (1978), em um estudo dos efeitos das condiçóes iniciais sobre o escoamento cisalhante livre axissimétrico, relataram o aumento de $K_{2} \operatorname{com} I_{0}$.

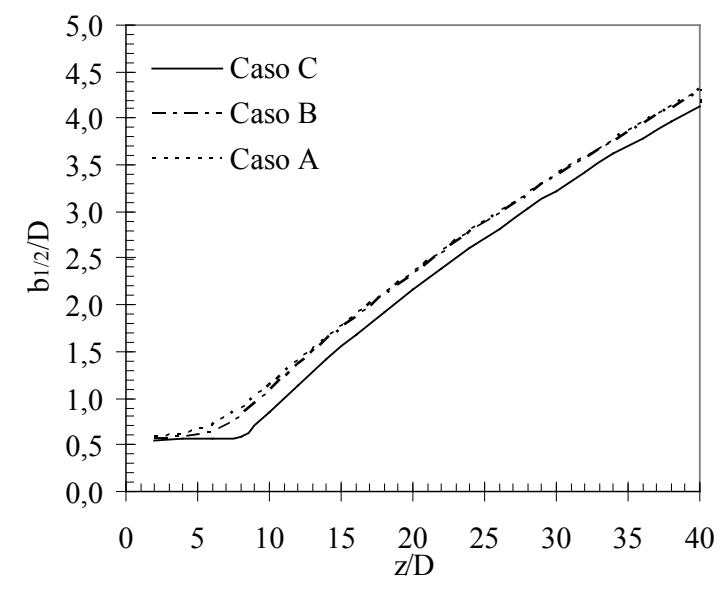

Figura 11: Evolução da meia largura do jato $b_{1 / 2}$ para diferentes intensidades de turbulência.

Tabela 2: Variação das origens virtuais $z_{01}$ e $z_{02}$, da taxa de decaimento do jato $\left(K_{1}\right)$ e da taxa de espalhamento do jato $\left(K_{2}\right)$.

\begin{tabular}{|c|c|c|c|c|c|c|c|c|}
\hline Caso & $\mathrm{I}_{0}(\%)$ & $J_{0,1}$ & $J_{0,2}$ & $\left.J_{0,2}\right|_{B, C} / J_{0,2}$ & $K 1$ & $z_{01}$ & $K 2$ & $z_{02}$ \\
\hline $\mathrm{A}$ & 17 & 0,0260 & 0,0268 & 1 & 5,708 & $-0,034$ & 0,113 & $-0,015$ \\
\hline $\mathrm{B}$ & 10 & 0,0260 & 0,0263 & 0,98 & 5,809 & $-0,032$ & 0,110 & $-0,011$ \\
\hline $\mathrm{C}$ & 1 & 0,0260 & 0,0260 & 0,97 & 5,815 & $-0,011$ & 0,108 & 0,010 \\
\hline
\end{tabular}




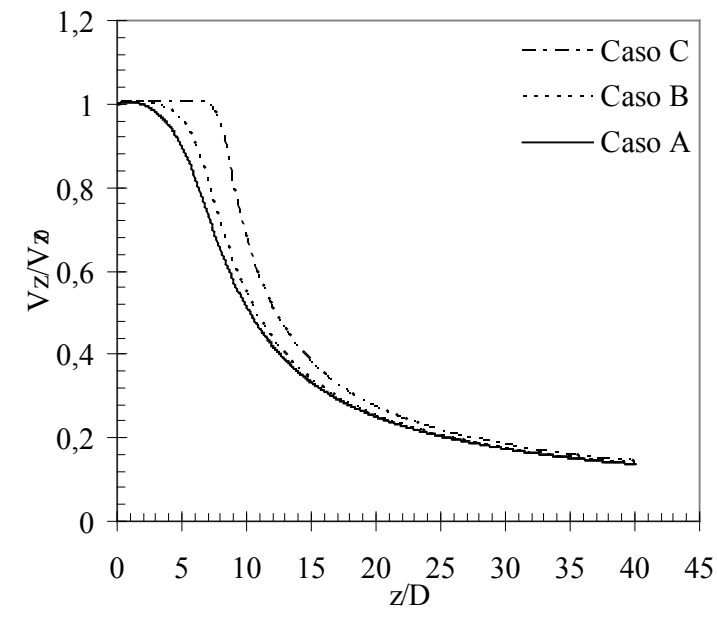

Figura 12: Evolução da velocidade na linha de centro do jato para diferentes intensidades de turbulência.

\subsection{PERFIL DE VELOCIDADE NA SAÍDA DO BOCAL}

As simulaçóes anteriores foram realizadas para um jato oriundo de um bocal com perfil de velocidade plano. Nesta etapa serão apresentados resultados de simulaçóes numéricas de um jato livre proveniente de um bocal com perfil de velocidade pistonado, típico de um escoamento tubular desenvolvido.

As simulações numéricas tiveram como objetivo reproduzir os dados experimentais de Frost e Jambunathan (1996) e de Mi et al. (2001b). A Tabela 3 sumariza as condiçóes dos jatos.

Tabela 3: Casos Simulados

\begin{tabular}{|c|c|c|c|}
\hline Caso & $\begin{array}{c}\text { Dados } \\
\text { Experimentais }\end{array}$ & $\mathrm{n}$ & $R e_{0}$ \\
\hline I & $\begin{array}{c}\text { Frost and } \\
\text { Jambunathan } \\
(1996)\end{array}$ & 6,5 & 22.500 \\
\hline II & Mi et al. (2001b) & 6,62 & 28.200 \\
\hline
\end{tabular}

Para ambos os casos, o perfil de velocidade pôde ser aproximado pela equaçáo $V z / V z_{\text {centro }}=$ $(1-2 r / D)^{1 / n}$ em que o expoente $n$ é dado na Tabela 3 .

Para os dois casos, a intensidade de turbulência na saída do bocal é dada na Figura 13. As condiçóes de contorno turbulentas para $k$ e $\varepsilon$ foram calculadas utilizando-se os valores da Figura 13 e as seguintes equaçóes:

$$
\left.k=\frac{3}{2}\left(U_{\text {avg }} I\right)^{2}\right), \varepsilon=C_{\mu}^{3 / 4} \frac{k^{3 / 2}}{l}, l=0.07 D_{b}
$$

em que o comprimento de escala $l$ representa a escala da turbulência, $I$ é a intensidade de turbulência, $D_{h}$ é o diâmetro hidráulico e $C_{\mu}$ é uma constante empírica (tipicamente 0.09).

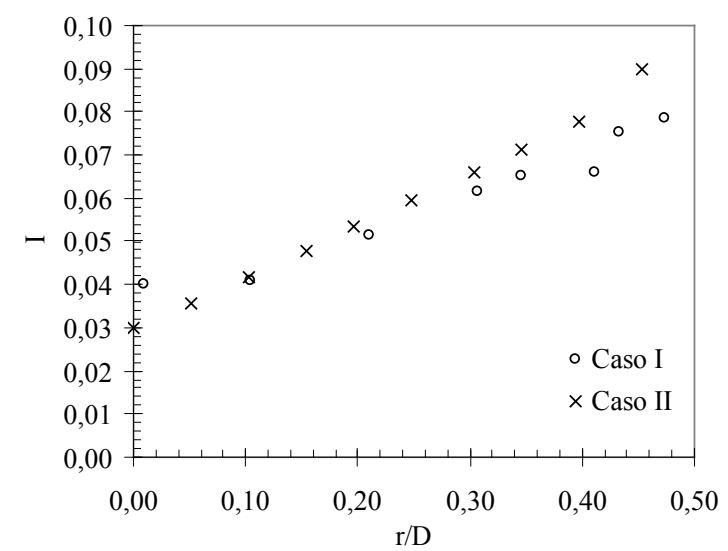

Figura 13: Perfil de intensidade de turbulência na saída do bocal.

É importante frisar que o Caso I e o caso da Seção 3 têm o mesmo número de Reynolds. Mi et al. (2001a) mostraram que, para um mesmo fluido e quando $\left(\operatorname{Re}_{0}\right)_{\text {desenvolvido }}$ é igual a $\left(\operatorname{Re}_{0}\right)_{\text {plano }}$, a quantidade de momento $(\mathrm{J})_{\text {desenvolvido }}$ é levemente maior $(1.2-2.8 \%)$ do que o $(\mathrm{J})_{\text {plano }}$. Em outras palavras, o mesmo número de $R e_{0}$ usado para os dois jatos fornece praticamente a mesma quantidade de momento. Neste trabalho, em particular, $(\mathrm{J})_{\text {plano }}=2.4 \%(\mathrm{~J})_{\text {desenvolvido }}$.

As Figuras 14 e 15 comparam os resultados das simulações numéricas com os dados experimentais dos Casos I e II, respectivamente.

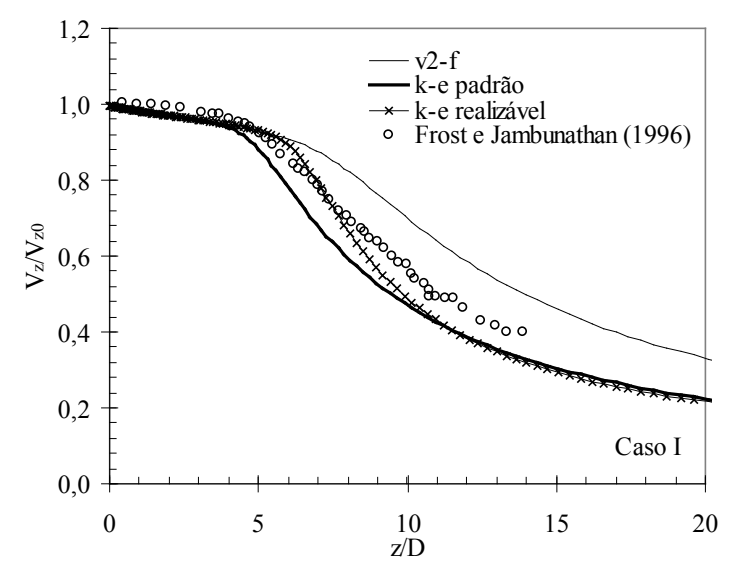

Figura 14. Caso I - Velocidade média axial normalizada no eixo de simetria axial versus distância adimensional a partir do bocal. 


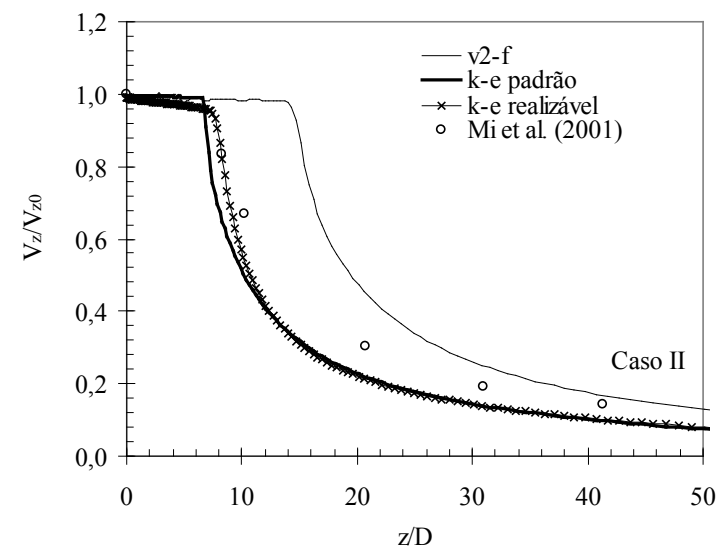

Figura 15. Caso II - Velocidade média axial normalizada no eixo de simetria axial versus distância adimensional a partir do bocal.

As Figuras 14 e 15 revelam que o modelo $k-\varepsilon$ realizável de Shih et al. (1995) não concorda satisfatoriamente com os dados experimentais. No sentido de explorar o desempenho dos modelos RANS, adicionalmente são mostrados resultados numéricos obtidos com outras duas versóes do modelo $k-\varepsilon$ : o modelo $k-\varepsilon$ padrão de Launder e Spalding (1972) e o de quatro equaçóes $v 2-f$ de Durbin (1995).

Observa-se que o perfil de velocidade experimental decai imediatamente após a saída do bocal, de modo que o comprimento da região núcleo potencial é muito pequeno e que os modelos de turbulência testados, em geral, proveram este decaimento.

Com exceção do modelo $v 2-f$ para o caso de Mi et al. (2001b), que previu uma longa regiáo potencial irreal, os modelos padráo e realizável previram o decaimento de velocidade maior do que os dados experimentais, indicando que estes modelos estimaram uma perda maior de momento com o ar estagnado.

Adicionalmente, resultados da previsão de cada modelo a respeito da intensidade de turbulência $(I)$ na linha de centro do jato são dados na Figura 16.

A intensidade de turbulência imediatamente após o bocal aumenta devido à camada de cisalhamento com o fluido vizinho. Esta camada cresce atingindo a linha de centro do jato, o que aumenta ainda mais a intensidade de turbulência.

Os três modelos previram esse comportamento, mas quantitativamente não concordam com os dados experimentais. No início, aproxima- damente para $\mathrm{z} / \mathrm{D}=4$, em que o efeito da camada cisalhante de mistura é pequeno, os três modelos possuem o mesmo perfil de intensidade de turbulência, mas inferior aos dados experimentais. Subsequentemente, os perfis divergem: o modelo $v 2-f$ apresenta uma intensidade de turbulência muito inferior ao experimental, o que justifica o decaimento lento da velocidade prevista por este modelo. Já os modelos $k-\varepsilon$ aproximam-se mais dos resultados experimentais: o $k-\varepsilon$ padráo prevê o máximo de intensidade de turbulência defasada e inferior à evidência experimental, enquanto o $k-\varepsilon$ realizável prevê corretamente a posiçáo de máxima intensidade.

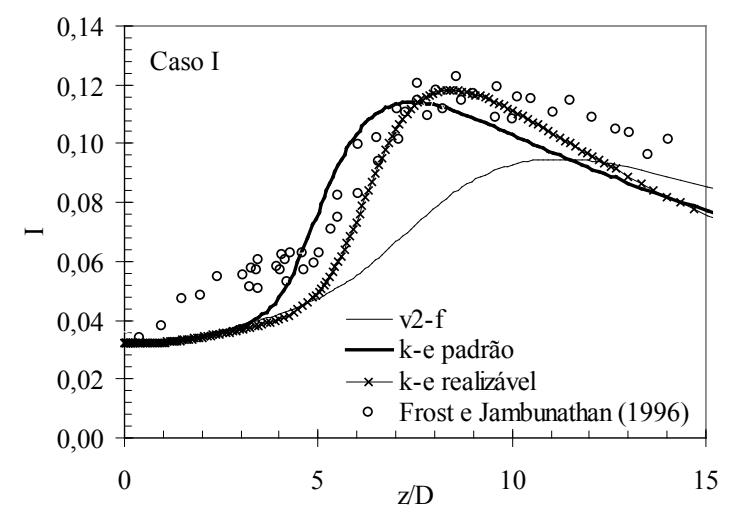

Figura 16. Comparação entre os dados experimentais e os três modelos analisados para a intensidade de turbulência na linha de centro do jato.

Sumarizando, ao contrário dos resultados da simulação do jato com perfil plano na saída apresentado na Seção 3, nenhum modelo de turbulência apresentou boa concordância com os dados experimentais para o jato com perfil desenvolvido na saída (Casos I e II). Alguns fatores como condiçôes de contorno e esquema numérico poderiam justificar esta discrepância, entretanto, náo obstante destes fatores, corrobora-se a conclusão de George e Davidson (2004), segundo os quais, por suas simplificaçóes físicas, os modelos RANS não são capazes de contabilizar corretamente as condiçóes físicas de origem do jato. Entretanto, conforme os resultados das Figuras 5 a 12,14 e 15 os modelos RANS podem contabilizar a dependência funcional com as condiçóes de origem na evolução do escoamento do jato. É importante ressaltar que em síntese, o que está sendo resolvido é um conjunto de equaçóes diferenciais parciais e a sua solução naturalmente depende das condiçóes de contorno. 
George e Davidson (2004) sugerem que as grandes escalas são as responsáveis pela preservação das condiçóes iniciais mais importantes e que a metodologia LES (simulação de grandes escalas), ao que parece, contém a física necessária para produzir uma assintótica dependência das condiçôes de origem.

Muitos autores propuseram o uso de funçóes ou novos valores para as constantes dos modelos de turbulência com o propósito de melhorar a previsão dos modelos quando o jato livre for plano ou axissimétrico, entretanto este procedimento reduz a ideia de generalidade do modelo.

Algumas funçôes são dadas em Launder et al. (1972), McGuirk and Rodi (1977) e Morse (1977).

A Figura 17 mostra resultados numéricos obtidos a partir dos três modelos de turbulência com suas constantes modificadas. No modelo $k-\varepsilon$ padrão a constante $C \varepsilon 1=1.44$ foi trocada para 1.60, como sugere Pope (1978) para simulaçóes de jatos circulares. Senesh e Babu (2005) relatam bons resultados numéricos com este valor para $C \varepsilon 1$ para um jato circular.

Para o modelo $v 2-f$, foi usado $C \varepsilon 1=1.3$ ao invés de $\mathrm{C} \varepsilon 1=1.4$, seguindo a sugestấo de Durbin (1995) para simulação de taxa espalhamento da camada de mistura. Para o $k-\varepsilon$ realizável foi usado $C \varepsilon 2=1.8$ ao invés de $C \varepsilon 2=1.9$.

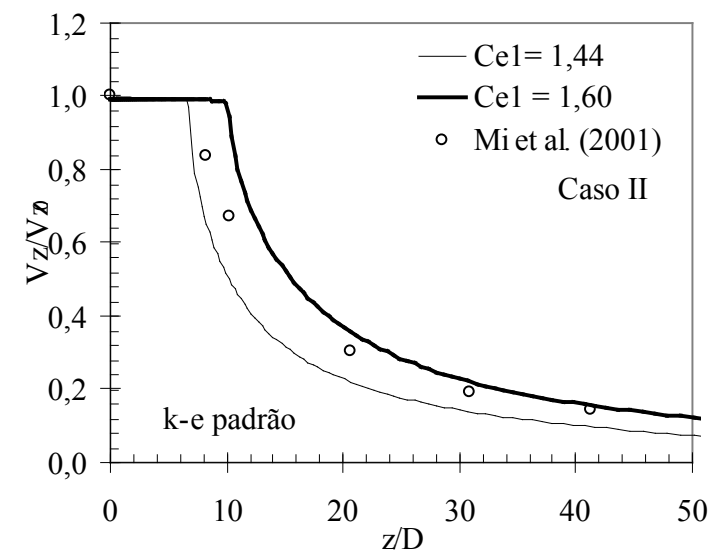

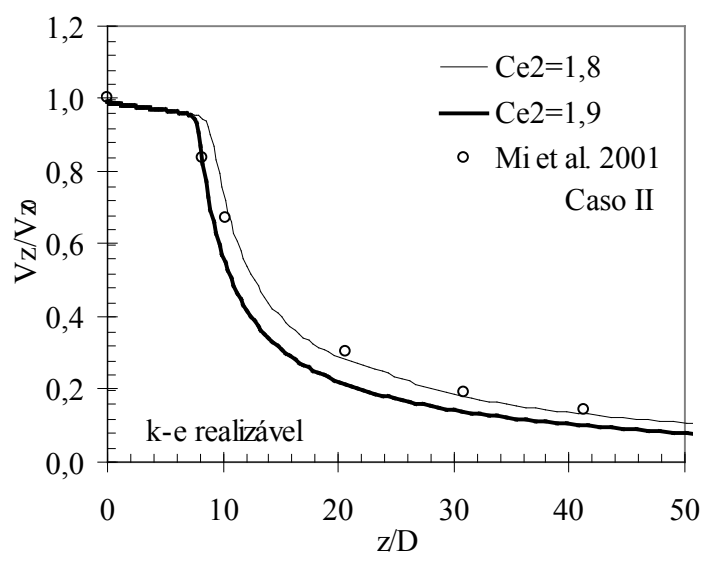

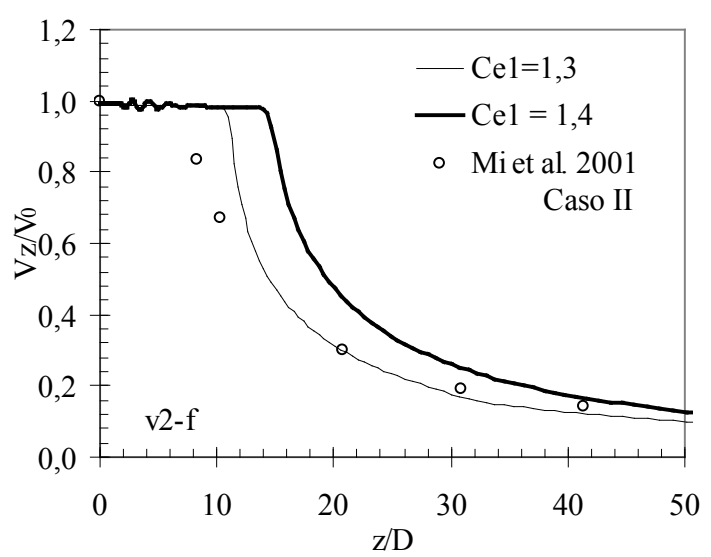

Figura 17: Caso II - Velocidade média axial normalizada no eixo de simetria axial versus distância adimensional a partir do bocal; resultados com os três modelos de turbulência com as constantes padrôes e modificadas.

Como esperado, a Figura 17 demonstra que as constantes dos modelos podem ser modificadas/calibradas para melhorar a previsão do modelo para um problema em especial. Entretanto, tal modificação ac-hoc limita o uso do modelo, podendo ser incerto em condiçóes inexploradas.

Como afirmam Jaw e Chen (1998) a necessidade de alterar as constantes na previsáo de escoamentos simples cisalhantes é uma decepção após o esforço envolvido na modelagem das equaçôes das tensôes de Reynolds, da energia cinética turbulenta e de sua dissipação.

Fundamentalmente, correções em um modelo não deveria prejudicar seu desempenho em situaçóes em que o modelo já mostrou-se adequado, tais correçóes deveriam apenas melhorar seu desempenho em situaçóes onde o comportamento é problemático e as várias correçôes deveriam ser unificadas para o seu uso em diversas condiçóes. 
Contudo, estes resultados proveem evidências adicionais de que as constantes dos modelos de turbulência não são universais. Nenhum conjunto de constantes seria absoluto, não haveria um conjunto único para todos os tipos de jatos. Como afirma George (1989), tal fato é pior do que a simples necessidade de constantes diferentes para a previsáo de jatos planos ou axissimétricos, uma vez que cada escoamento requererá uma multiplicidade de constantes para contabilizar as diferentes condiçôes de origem.

Contudo, na maioria das aplicações de engenharia, não é impeditivo o uso dos modelos single point RANS, quando utilizados com critério. Esta abordagem há mais de três décadas provê resultados importantes nas mais diversas áreas.

Outras metodologias como LES e DNS (simulação numérica direta) requerem relativa maior memória, tempo e capacidade computacional, tornando-as, vezes pouco atraentes no sentido de praticidade para aplicaçóes de engenharia.

\section{CONCLUSÃO}

No presente trabalho, o problema de jato livre turbulento com diferentes condiçóes de origem foi simulado numericamente. A partir dos resultados obtidos, foi possível obter as seguintes conclusóes:

- parâmetros $z_{01}, K_{1}, z_{02}$ e $K_{2}$ apresentaram maior variação para baixos números de Reynolds $3.000 £ R e_{0} £ 10.000$;

- a origem virtual $z_{02}$ não apresentou a variação sistemática para a faixa explorada de $10.000 £ R e_{0} £ 70.000$;

- os parâmetros $z_{01}, K_{1}$ e $K_{2}$ apresentaram variação assintótica com $R e_{0}$ para altos valores deste;

- a intensidade de turbulência do fluido, no plano de saída do bocal, apresentou forte influência nas regiôes núcleo potencial e completamente desenvolvida. Para os valores explorados de $I_{0} 1 \%, 10 \%$ e $17 \%$ houve redução do comprimento da regiáo núcleo potencial. Na regiáo completamente desenvolvida, os valores dos parâmetros $z_{01}, z_{02} \mathrm{e}$ $K 1$ reduziram com o aumento de $I_{0}$;

- resultados obtidos no presente trabalho evidenciam que a regiáo completamente desenvolvida não é dependente apenas da quantidade de momento na saída do bocal
$\left(J_{0}\right)$; consequentemente, os jatos atingem diferentes estados autossimilares em funçáo dos detalhes de sua origem;

- os modelos RANS de turbulência podem até contabilizar a dependência funcional com as condiçóes de origem na evolução do escoamento do jato, entretanto os resultados do presente trabalho corroboram a afirmação de Taulbee (1989), George (1989) e George e Davidson (2004): por suas simplificaçôes físicas, os modelos RANS não são capazes de avaliar corretamente os efeitos das condiçóes de origem do jato.

\section{REFERÊNCIAS}

BECKER, H. A., HOTTEL, H. C., WILLIAMS, G. C. (1967). The nozzle fluid concentration field of the round turbulent jet. J. Fluid Mech, vol.30, pp. 285-303.

CHANG, I. Unsteady-State Underexpanded Jet Flows, AIAA/ASME/SAE/ASEE Joint Propulsion Conference and Exhibit 7-10 July 2002, Indianapolis, Indiana, p. 1-12.

Chen, C. J., Jaw, S. Y. Fundamentals of Turbulence Modeling. Copyright (C) 1998 Taylor \& Francis

DAHM, W. J. A., DIMOTAKIS, P. E. (1990). Mixing at Large Schmidt Number in the SelfSimilar Far Field of Turbulent Jets. J. Fluid Mech., vol. 217, pp. 299-330.

DOWLING, D. R., DIMOTAKIS, P. E. (1990). Similarity of the concentration field of gas-phase turbulent jets. J. Fluid Mech, vol. 218, pp. 109141.

DOWLING, D. R.,DIMOTAKIS P. E. (1990). Similarity of the concentration field of gas-phase turbulent jets. J. Fluid Mech. vol.218, pp.109141.

DURBIN, P. A. (1995). Separated Flow Computations with the $k-\varepsilon-v^{2}$ Model. AIAA J., vol.33, n.4, pp.659-664.

FROST, S. A., JAMBUNATHAN, K. (1996). Effect of Nozzle Geometry and Semi-confinement 
on the Potential Core of a Turbulent Axisymmetric Free Jet. Int. Comm. Heat Mass Transfer, vol. 23, n.2, pp. 155-162.

GEORGE, W. K. (1989). The self-similarity of turbulent flows and its relation to initial conditions and coherent structures. In Recent Advances in Turbulence (ed. R. E. A. Arndt and W. K. George), pp. 39-73.

GEORGE, W. K.; DAVIDSON L. (2004). Role of Initial Conditions in Establishing Asymptotic Flow Behavior. AIAA Journal, vol. 42, n. 3, pp. 438-446.

GRANDMAISON, E.W.; RATHGEBER, D. E.; BECKER, H. A. (1982). Some Characteristics of Concentration Fluctuations in Free Turbulent Jets. Can. J. Chem. Eng. vol.60, pp.212-219.

HUSSAIN, A. K.M. F., ZEDAN,M. F. (1978). Effects of the Initial Conditions on the Axisymmetric Free Shear Layer: Effect of the Initial Fluctuation Level. Physics of Fluids, vol.21, pp. 1475-1481.

HUSSEIN H. J., CAPP S. P., GEORGE W. K. (1994). Velocity Measurements in a High Reynolds Number, Momentum-Conserving Axisymmetric Turbulent Jet. J. Fluid Mech., vol. 258, pp.31-75.

JOHANSSON, P. B. V., GEORGE, W. K., GOURLAY, M. J. (2003). Equilibrium Similarity, Effects of Initial Conditions and Local Reynolds Number on the Axisymmetric Wake. Physics of Fluids $A$, vol.15, n.3, p.603-617.

KEYSAR, L., DEGANI D. (2004). Numerical Investigation of Axisymmetric Compressible Turbulent Jets, $42^{\text {nd }}$ AIAA Aerospace Sciences Meeting and Exhibit, Reno, Nevada.

KLEIN M., SADIKI A., JANICKA J. (2003). Investigation of the Influence of the Reynolds Number on a Plane Jet using Direct Numerical Simulation. I. J. Heat and Fluid Flow vol.24, pp.785-794.

KLEIN, M., SADIKI, A., JANICKA J. (2003). Investigation of the influence of the Reynolds number on a plane jet using direct numerical simulation. I. J. Heat and Fluid Flow, vol.24, pp.785-794.

LAUNDER, B. E., SPALDING, D. B. (1972). Lectures in Mathematical Models of Turbulence, Academic Press, London, England.

MCGUIRK, J. J., RODI, W. (1977). The Calculation of Three-Dimensional Free Jets, Symposium on Turbulent Shear Flows, Pennsylvania State University.

MCQUAID, J.; WRIGHT, W. (1974). Turbulence Measurements with Hot-Wire Anemometry in Nonhomogeneous Jets. Int. J. Heat Mass Transfer, vol.17, pp.341-349.

MI, J., NATHAN, G.J., NOBES, D.S., (2001b). Mixing Characteristics of Axisymmetric Free Jets From a Contoured Nozzle, an Orifice Plate and a Pipe, J. Fluids Engineering, ASME, vol. 123, pp. 878-883.

MI, J., NOBIS, D. S., AND NATHAN. G. (2001a) Influence of Jet Exit Conditions on the Passive Scalar Field of an Axisymmetric Free Jet. Journal of Fluid Mechanics, vol. 432, pp. 91-125.

MORSE, A. P., (1977), Axisymmetric Turbulent Shear Flows with and without Swirl, Ph. D. Thesis, London University, England.

MOSER, R. D., ROGERS, M. M., EWING, D. W. (1998). Self-Similarity of Time-Evolving Plane Wakes, Journal of Fluid Mechanics, vol. 367, pp. 255-289..

NAMER, I., ÖTÜGEN, M., (1988). Velocity Measurements in a Plane Turbulent Air Jet at Moderate Reynolds Numbers. Exp. Fluids, vol. 6, pp. 387-399.

PANCHAPAKESAN N. R, LUMLEY J. L. (1993). Turbulence Measurements in Axisymmetric Jets of Air and Helium. Part 1. Air jet, J. Fluid Mech., vol. 246, 197-223.

PITTS, W. M. (1991a). Effects of global density ratio on the centerline mixing behavior of axisymmetric turbulent jets. Exps. Fluids, vol. 11, pp.125-134. 
PITTS, W. M. (1991b). Reynolds number effects on the centerline mixing behavior of axisymmetric turbulent jets. Exps. Fluids, vol. 11, pp. 135144.

POPE, S. B., (1978). An Explanation of the Round Jet/Plane Jet Anomaly. AIAA J., vol. 3, pp. 279-281.

RICHARDS, C. D.; PITTS, W. M. (1993). Global density effects on the self-preservation behaviour of turbulent free jets. J. Fluid Mech, vol. 245, pp. 417-435.

RODI, W. (1976). A New Algebraic Relation for Calculating the Reynolds Stresses. Z.Argew. Math. Mech, vol. 56, pp. 219-221.

SCHILICHTING, H. (1979). Boundary Layer Theory, Mc-Graw-Hill, New York.

SENESH, K., BABU, V. (2005). Numerical Simulation of Subsonic and Supersonic Jets. $11^{\text {th }}$ AIAA/CEAS Aeroacoutics Conference.

SHIH, T.-H., LIOU W. W., SHABBIR A., YANG Z., ZHU J. (1995). A New $k-\varepsilon$ Eddy-Viscosity Model for High Reynolds Number TurbulentLows-Model Development and Validation. Computers Fluids, vol.24, p.227-238.

SO, R. M., ZHU C., ÖTÜGEN, HWANG, B. C. (1990). Some Measurements in a Binary Gas
Jet. Exps. Fluids, vol.9, 273-284, 1990.

STANLEY, S. A., SARKAR S. (2000). Influence of Nozzle Conditions and Discrete Forcing on Turbulent Planar Jets. AIAA, vol. 38, n. 9.

STRÖHER, G. R. (2008). Avaliação de Modelos de Turbulência para Jatos Livres Circulares. $176 f$. Tese (Doutorado em Ciências) - Instituto Tecnológica de Aeronáutica, São José dos Campos.

TANAKA, T., TANAKA, E. (1976). Experimental Study of a Radial Turbulent Jet. Bulletim of the JSME, vol. 19. pp. 792-799.

TAUlBEE, D. B. (1989). Reynolds Stress Models Applied to Turbulent Jets,"Advances in Turbulence, edited by W. K. George and R. E. A. Arndt,Hemisphere, New York, pp. 39-73.

TOWNSEND, A. A. (1976). The Structure of Turbulent Shear Flow, $2^{\text {nd }}$ edition. Cambridge University Press.

VANDOORMAAL, J. P., AND RAITHBY G. D. (1984). Enhancements of the SIMPLE Method for Predicting Incompressible Fluid Flows. Numer. Heat Transfer, vol. 7, pp.147-163.

WYGNANSKI, I., H. FIELDLER. (1969). Some Measurements in the Self-Preserving jet. J. Fluid Mech., vol.38, pp.577-612. 